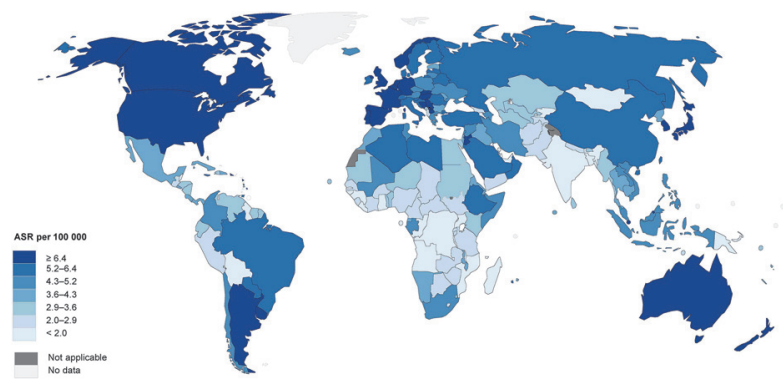

Abstract IDDF2021-ABS-0181 Figure 1 Global age-standardised incidence of early-onset colorectal cancer in 2020. ASR: agestandardised rate; Data-source: GLOBOCAN 2020 Graph production: IARC (http://gco.iarc.fr/today) World Health Organization

Background This study aimed to investigate the incidence of and the risk factors associated with early-onset colorectal cancer (CRC) using data from 185 countries.

Methods The Global Cancer Observatory (GLOBOCAN) was retrieved for the age-standardised rates (ASR) per 100000 for incidence of CRC in 2020 among individuals aged 15 to 49 years old. The prevalence of different risk factors in 2020 among the young population for each country was estimated using joinpoint regression. The associations between risk factors and incidence were measured using beta coefficients $(\beta)$ with $95 \%$ confidence intervals $(\mathrm{CI})$ by multivariable linear regression adjusting for Gross Domestic Product (GDP) and Human Development Index (HDI).

Results In 2020, the total number of new early-onset CRC was 187454 (9.7\% of CRC for all ages), with an ASR of 4.6 (IDDF2021-ABS-0181 Figure 1. Global standardised incidence of early-onset colorectal cancer in 2020). The highest ASR incidence of early-onset CRC was in males (4.8 vs. 4.4 in females); Australia and New Zealand (11.0), Northern America (9.9), Western Europe (7.2); and regions of Very High HDI (7.3) and high HDI (5.1). Countries with a higher incidence of early-onset CRC had a higher prevalence of smoking $(\beta=0.06$, CI $0.02-0.10)$, alcohol drinking $(\beta=0.10$, CI $0.03-$ $0.16)$, unhealthy dietary $(\beta=0.04$, CI $0.02-0.07)$, and lipid disorders $(\beta=0.08$, CI $0.05-0.11)$ among the younger population. Conclusions There was a substantial burden of early-onset CRC in 2020, with higher incidence observed in males and developed regions. The associated risk factors for early-onset CRC included smoking, alcohol drinking, unhealthy dietary habits, and lipid disorders. More intensive lifestyle modifications, chronic disease management, and population-based screening for selected younger individuals are recommended to prevent early-onset CRC.

\section{IDDF2021-ABS-0182 EFFECTIVENESS OF COMPLEX INTERVENTIONS FOR REDUCING 30-DAY HOSPITAL READMISSIONS AMONG COLORECTAL SURGERY PATIENTS: OVERVIEW OF SYSTEMATIC REVIEWS AND NETWORK META-ANALYSIS}

${ }^{1}$ Claire Chenwen Zhong*, 'Charlene Hoi Lam Wong, 'Anna Kam Nam Tsoi, 'William Kwok Wai Cheung, 'Eng-kiong Yeoh, ${ }^{1}$ Chi Tim Hung, 'Benjamin Hon Kei Yip, ${ }^{1}$ Eliza Lai Yi Wong, ${ }^{1}$ Samuel Yeung Shan Wong, ${ }^{1,2}$ Vincent Chi Ho Chung. 'Jockey Club School of Public Health and Primary Care, The Chinese University of Hong Kong, Hong Kong; ${ }^{2}$ School of Chinese Medicine, The Chinese University of Hong Kong, Hong Kong

10.1136/gutjnl-2021-IDDF.170
Background To evaluate the comparative effectiveness of different complex interventions for reducing 30-day hospital readmissions and length of stay among colorectal surgery patients via an overview of systematic reviews (SRs) and network meta-analysis (NMA).

Methods We searched five databases for eligible SRs of randomized controlled trials (RCTs) and conducted an additional search for updated RCTs published from databases' inception to August 2020. We conducted random-effect pairwise meta-analyses using pooled risk ratios (RRs) with 95\% confidence intervals (CIs), and mean differences (MDs) with 95\% CIs to quantify the effect of complex interventions for

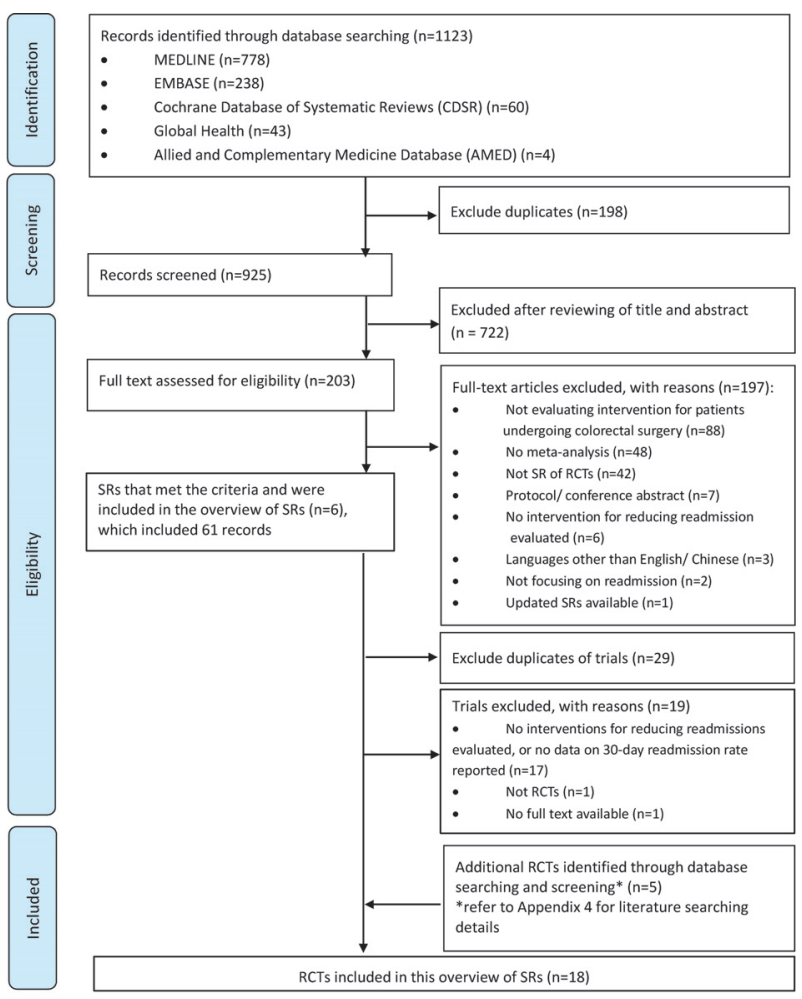

Abstract IDDF2021-ABS-0182 Figure 1 Flowchart of literature search and selection for systematic reviews and randomized controlled trials

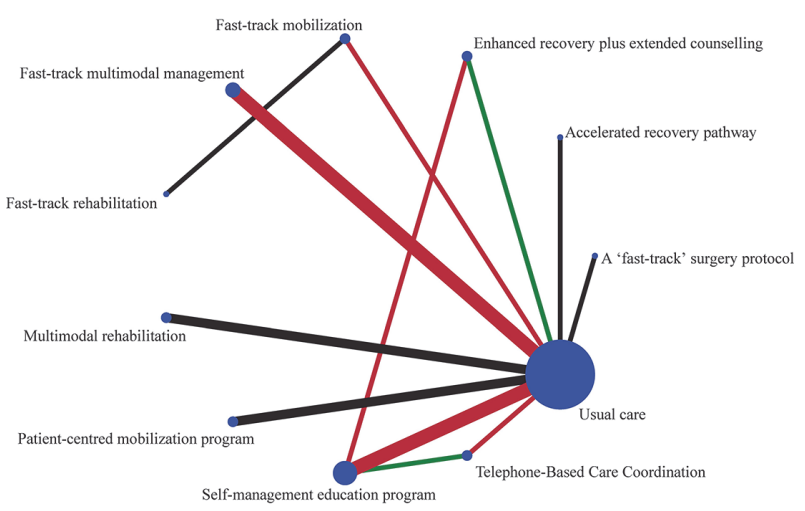

Abstract IDDF2021-ABS-0182 Figure 2 Network plot of comparisons among 10 different complex interactions and usual care in the network meta-analysis for reducing 30-day hospital readmissions among colorectal surgery patients 
binary and continuous outcomes, respectively. We performed NMA to evaluate comparative effectiveness among different complex interventions. Primary outcome was 30-day hospital readmissions, while secondary outcomes were length of stay, 30-day mortality and 30-day emergency department (ED) visits.

Results A total of six SRs and18 RCTs ( $n=3,071)$ assessing ten different complex interventions were included (IDDF2021-ABS-0182 Figure 1. Flowchart). Results of pairwise meta-analyses showed no significant difference between complex interventions and controls in reducing 30-day hospital readmissions (pooled RR: 0.93; 95\% CI: 0.73, 1.18; 16 RCTs; moderate quality of evidence), 30-day mortality (pooled RR: 1.30; 95\% CI: 0.59, 2.82; 9 RCTs; low quality of evidence) and 30-day ED visits (pooled RR: 0.81; 95\% CI: $0.55,1.21$; 2 RCTs; moderate quality of evidence). However, pairwise meta-analysis results showed that complex interventions significantly reduced length of stay (pooled MD: -2.12 days; 95\% CI: $-2.79,-1.44$ days; 14 RCTs; very low quality of evidence). NMA results indicated that there was no significant difference across different complex interventions on reducing 30-day hospital readmissions (IDDF2021-ABS-0182 Figure 2. Network plot). Nonetheless, fast-track rehabilitation might be the most effective intervention for reducing the length of stay, as supported by low certainty of evidence.

Conclusions NMA showed that complex interventions may not differ from usual care in reducing 30-day hospital readmissions among colorectal surgery patients. However, fast-track rehabilitation may have the highest potential for reducing the length of stay. The benefits of fast-track rehabilitation for colorectal surgery patients may be different across different health systems. Policymakers may consider contextual factors carefully before implementation.

\section{IDDF2021-ABS-0188 WORLDWIDE INCIDENCE AND LIFESTYLE RISK FACTORS OF GASTRIC CANCER AMONG YOUNG ADULTS: A GLOBAL STUDY}

${ }^{1} J u n j i e ~ H u a n g *,{ }^{1}$ Alfonse Ngai, ${ }^{2}$ Veeleah Lok, ${ }^{3}$ Xianjing Liu, ${ }^{4}$ Lin Zhang, ${ }^{5}$ Jinqiu Yuan, ${ }^{6}$ Wanghong Xu, ${ }^{7}$ Zhi-Jie Zheng, 'Martin CS Wong. 'Jockey Club School of Public Health and Primary Care, Faculty of Medicine, Chinese University of Hong Kong, Hong Kong; ${ }^{2}$ Department of Global Public Health, Karolinska Institute, Karolinska University Hospital, Stockholm, Sweden; ${ }^{3}$ Department of Radiology and Medical Informatics, Erasmus University Medical Centre, Rotterdam, Netherlands; ${ }^{4}$ Melbourne School of Population and Global Health, The University of Melbourne, Victoria, Australia; ${ }^{5}$ Clinical Research Centre, The Seventh Affiliated Hospital, Sun Yat-sen University, Guangdong, China; ${ }^{6}$ School of Public Health, Fudan University, Shanghai, China; ${ }^{7}$ Department of Global Health, School of Public Health, Peking University, Beijing, China

\subsection{6/gutjpl-2021-IDDF.171}

Background The purpose of this study is to investigate the incidence and lifestyle risk factors for gastric cancer in younger individuals aged 15-49 years old using global health and national cancer registry data.

Methods We retrieved the Global Cancer Observatory (GLOBOCAN) 2020 for age-standardised incidence (ASI) per 100 000 of gastric cancer in young individuals across 185 countries. We estimated the prevalence of lifestyle risk factors (smoking, alcohol drinking, and dietary habits) among the younger adults by joinpoint regression. We conducted the multivariable linear regression to examine the associations between lifestyle risk factors and incidence whilst adjusting for gross domestic products (GDP) per capita for each country.

Results The total number of new cases of young-onset gastric cancer was 100687 in 2020, with an ASI of 2.5 (IDDF2021ABS-0188 Figure 1. Worldwide incidence of young-onset gastric cancer in 2020). The highest ASI was in males (2.9 vs. 2.1 in females); Eastern Asia (3.7), Central America (2.4),

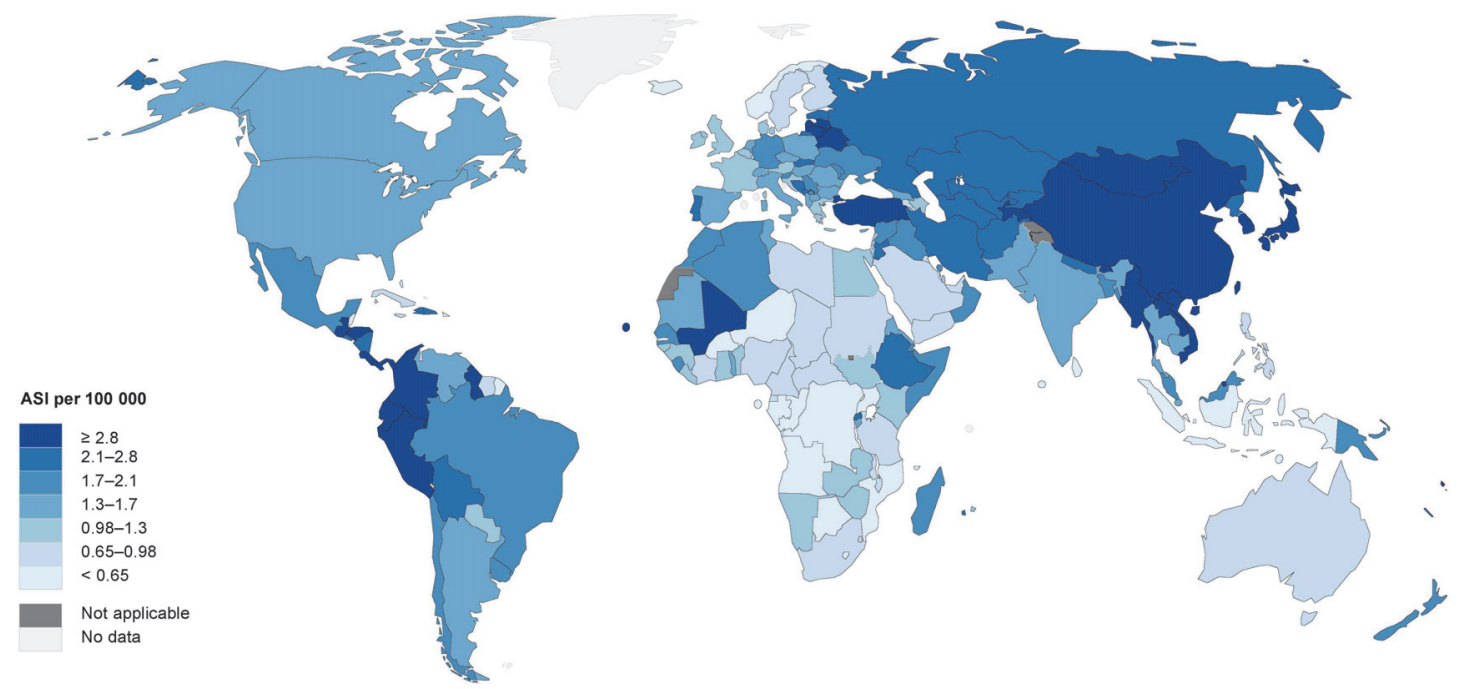

Abstract IDDF2021-ABS-0188 Figure 1 Worldwide incidence of young-onset gastric cancer in 2020. ASI, age-standardised incidence; Data source: GLOBOCAN 2020 Graph production: IARC (http://gco.iarc.fr/today) World Health Organization 